\title{
Las prácticas de citación como interpretantes semióticos de acreditación de saberes locales en astronomía: México 1952-1972
}

\author{
Citation practices as semiotic interpretants \\ of certification of local knowledge in \\ astronomy: Mexico 1952-1972
}

\author{
Francisco COLLAZO-REYES² \\ Xochitl FLORES-VARGAS ${ }^{3}$ \\ Mitzi Lizeth MUÑOZ-GARCÍA ${ }^{3}$ \\ Miguel Ángel PÉREZ-ANGÓN²
}

\section{Resumen}

Se estudia la literatura científica en el área de astronomía publicada en México. Para ello se utilizan los textos publicados en el Boletín de los Observatorios de Tonantzintla y Tacubaya, durante un periodo de cambios importantes en la producción y publicación de conocimientos científicos en México, 1952-1972. Se busca identificar, en los textos científicos del Boletín, marcas de afiliación del texto local a los patrones de la comunicación científica moderna. Para ello revisamos las prácticas de citación seguidas en las publicaciones, particularmente las funciones de comunicación de las referencias como elementos retóricos de persuasión, en el proceso de formación del texto científico. Se utiliza una metodología de análisis semiótico basada en el modelo tríadico de formación de conceptos. A través de esta metodología fue posible mostrar nuevos roles de los textos del Boletín en el sistema de la comunicación científica internacional. Interpretantes semióticos que dan cuenta de dos funciones de comunicación adquiridas por los textos: una como fuentes acreditadas y otra como fuentes acreditantes, en el proceso mutuo de certificación de conocimientos seguido en las revistas científicas.

Palabras ilave: Análisis semiótico. Astronomia. Bibliografías citadas. Intertextualidad. México. Periódico científico.

\begin{abstract}
We studied the scientific literature in the field of astronomy published in the Mexican Journal, Boletin de los Observatorios de Tonantzintla y Tacubaya, from 1952 to 1972. We analyzed the articles published during this important period of great changes in the production and dissemination of scientific knowledge. Our goal is to identify affiliation practices in the patterns of scientific communication. The characteristics of citation practices were reviewed, particularly the communication functions of references as rhetorical elements of persuasion. Semiotic analysis was used, particularly the triadic model of concept formation. Accordingly, it was possible to demonstrate new roles of the Boletín articles within the framework of the system of international communication. Semiotic interpretants reveal two communication functions acquired by the texts: in the first case, sources are accredited, and the second case, sources are accrediting, in a mutual process of certification of knowledge found in scientific journals.
\end{abstract}

Keywords: Semiotic analysis. Astronomy. Citation bibliographies. Intertextuality. México. Scientific journals.

\footnotetext{
1 Trabajo presentado en el VII Seminario Internacional sobre Estudios Cuantitativos y Cualitativos de la Ciencia y la Tecnología "Prof. Gilberto Sotolongo Aguilar"en XIII Congreso Internacional de Información - INFO'2014. Habana, Cuba.

2 Centro de Investigación y de Estudios Avanzados, Departamento de Física, Biblioteca de Ciencias Exactas. Av. Instituto Politecnico Nacional 2508, Colonia San Pedro Zacatenco, 07360, Ciudad de México, México. Correspondencia a nombre de/Correspondence to: F. COLLAZO-REYES. E-mail: $<$ fcollazo@fis.cinvestav.mx>.

${ }^{3}$ Centro de Investigación y de Estudios Avanzados, Biblioteca de Ciencias Exactas, Area de Bibliometría. Ciudad de México, México.

Recibido el día 10/6/2014 y aceptado para su publicación el 12/9/2014.
} 


\section{Introducción}

La intertextualidad referencial (Porter, 1986; Haberer, 2007) forma parte de la naturaleza del discurso científico (Bazerman, 1988a, 1993). Es un componente bibliográfico que ha incrementado su importancia en el proceso de evolución del género científico moderno (Bazerman, 1988b) y lo ha distinguido de otros estilos literarios. Esta importancia se puede observar en la tendencia generalizada de incremento, en el tiempo, del número de referencias por trabajo, principalmente en las ciencias exactas, naturales y las ingenierías. Este hecho ha estado acompañado de distintas interpretaciones sobre las funciones de las referencias en el texto científico. La literatura en bibliometría/cienciometría da cuenta de este debate (Cronin, 1981; Cozzens, 1989; Bornmann \& Daniel, 2008).

Los recursos argumentativos de estas propuestas han estado influenciados, históricamente, por dos concepciones distintas, ambas relacionadas con el desarrollo de la ciencia. Una influenciada por las teorías de Merton (1968). Este considera la ciencia como una institución normativa gobernada por un sistema interno de normas, recompensas y sanciones, que guían y constriñen las acciones de los científicos (Cronin, 1984; White, 2004). Las citas, como parte de este sistema, son consideradas como eventos de reconocimiento o recompensa. Existe una abundante literatura y consenso sobre el concepto de la cita como una relación de reconocimiento entre el documento que cita (citante) y los documentos citados. A partir de esta interpretación se han desarrollado las concepciones dominantes sobre las funciones de las citas, como es el caso del factor de impacto en todas las modalidades (Cronin, 1984).

Otras propuestas conciben la ciencia como prácticas socialmente construidas (Knorr-Cetina, 1981; Latour, 1987). Aquí el uso de las referencias está gobernado por una función de persuasión (Gilbert, 1977; Paul, 2000; Moed, 2005). Como componentes del arte retórico de la persuasión (Restrepo Forero, 2004; Carmona Sandoval, 2013). Bajo esta concepción se asume que una n. 14 de las mayores motivaciones de citar está conectada con el deseo de persuadir lectores (MacRoberts, M.H. \& MacRoberts, B.R., 1987). En esta interpretación de las referencias como dispositivos retóricos para persuadir (Restrepo Forero, 2004; Carmona Sandoval, 2013), el autor tiende a citar trabajos importantes o autorizados, de tal manera que el trabajo que los cita participa de su autoridad (Gilbert, 1977). Esta concepción de las referencias ha permanecido un poco olvidada (White, 2004; Davis, 2009). Sin embargo, el concepto de persuasión resulta más adecuado para los trabajos de corte histórico, como el que aquí realizamos. Creemos que los cambios en la formación de los patrones de citación, como parte del proceso histórico de formación del texto científico, forman parte de un escenario mayor. La creación de condiciones que hacen posible la producción de un sentido (Beuchot, 2004) de filiación de la ciencia local a los cánones de la ciencia internacional. También, que es posible caracterizar este sentido de filiación, a través del estudio de las relaciones entre textos (intertextualidad referencial), como signos de acreditación, entre textos, que en la medida que se repiten, significan la relación entre discursos con contenidos afines.

En los procesos de conformación de los sistemas de comunicación científica locales, basados en las fuentes e idioma doméstico, las prácticas de citación moderna tardaron en formar parte de la estructura de los contenidos y el estilo argumentativo de los textos científicos (Luna-Morales et al., 2009). En México, los Anuarios de los Observatorios de Tacubaya y de Chapultepec, Las Memorias de la Sociedad Científica Antonio Alzate, y El Boletín de la Sociedad Astronómica de México, cubren el periodo histórico más largo de conocimientos publicados en astronomía, 1881-1950 (González-Tapia \& Muñoz-García, 2013). El cuerpo de literatura acumulado en estas revistas contiene los temas, tipos de investigación, estilos, idioma, métodos e instrumental científico, utilizados en este tipo de investigación observacional. Este periodo, está dominado por temáticas relacionadas con la astronomía clásica o astronomía de posición y las observaciones relacionadas con la elaboración de la Carta y el Catálogo astrofotográfico del cielo de México, como parte de un proyecto internacional (Bok, 1983; Pismis, 1987; Bartolucci, 1991, 2000).

Estas prácticas de investigación dieron lugar a las estructuras de un sistema de publicación y comunicación científica local. Dependiente de los criterios mínimos seguidos para publicar en las fuentes mencionadas. 
Predominó un estilo de contenidos con funciones de comunicación orientadas a la divulgación de conocimientos astronómicos generales sobre diversos aspectos: cometas, eclipses, luna, cielo, sol, planetas (Júpiter, Marte, Saturno), estrellas, observatorios, constelaciones, entre otros; así como desarrollo de trabajos sobre aspectos aplicados: determinar posiciones geográficas específicas, elaborar mapas e informes periódicos sobre datos astronómicos reportados por mes, así como reportes sobre fenómenos astronómicos importantes (González-Tapia \& Muñoz-García, 2013). En el desarrollo de estos contenidos muy pocas veces se recurría a las referencias y éstas no forman parte sustantiva del estilo argumentativo de los textos. Los primeros trabajos publicados del área en revistas internacionales (Transactions-American Geophysical Union y Astronomical Journal), tienen estas mismas características: tratan aspectos descriptivos referentes al trabajo administrativo y al equipo utilizado en los observatorios, no incluyen referencias (Gallo, 1939; RiveraTerrazas, 1950). Los primeros antecedentes de prácticas modernas de citación, en la astronomía generada en México, se encuentran en un trabajo escrito por Munch (1947) con adscripción al Observatorio de Tacubaya. Es un trabajo teórico que incluye 8 referencias y ha recibido 19 citas bibliográficas.

Históricamente la vía común de acreditación internacional del conocimiento generado localmente ha sido a través de la publicación arbitrada en revistas reconocidas en la corriente principal de cada área. Aquí nos referimos a prácticas científicas desarrolladas y difundidas en las condiciones adversas de un contexto local-local (Cueto, 1997). Se trata de los conocimientos generados en observatorios locales y publicados en una revista local, el Boletín de los Observatorios de Tonantzintla y Tacubaya (BOTT). Una revista que en su momento de vigencia, 1952-1972, estuvo relegada del Science Citation Index (SCI), aunque este índice da cuenta de las citas recibidas, por trabajos del BOTT, de revistas cubiertas en este índice. Con el tiempo ha adquirido mayor visibilidad internacional aún después que dejó de publicarse. Actualmente la colección completa de textos se encuentra disponible en línea en la Universidad Nacional Autónoma de México (UNAM) <http:// www.astroscu.unam.mx/bott/> y en el sistema Astrophysic Data System (ADS) / National Aeronautics and
Space Administration (NASA) < http://adsabs.harvard.edu/ bib_abs.html>. En el año internacional de la astronomía, 2009, la comunidad científica mexicana en astronomía organizó un congreso internacional "Highlights of Boletín de los Observatorios de Tonantzintla y Tacubaya" (TorresPeimbert et al., 2011). Tuvo como finalidad revisar el rol del BOTT en el desarrollo de la astronomía moderna en México. Los trabajos presentados ofrecen una revisión de la producción histórica del Boletín, principalmente los trabajos más citados. Se abordan aspectos referentes a los resultados, las contribuciones, relevancia, temas iniciales, circunstancias del contexto, instrumental científico y técnicas utilizadas; a la luz de los más recientes desarrollos en el campo (López-Cruz, 2011; CornejoRodríguez, 2011; Abt, 2011 a, 2011b).

En este trabajo, identificamos y revisamos las estructuras de relaciones de comunicación científica, desarrolladas en las prácticas de investigación y publicación de los trabajos del BOTT. Para ello utilizamos las referencias hechas y las citas recibidas en los textos. Lo anterior con el fin de caracterizar estos flujos de comunicación, como un método de aproximación al proceso de acreditación de los textos del Boletín en los distintos discursos a que pertenecen las referencias hechas y los discursos de donde proceden las citas recibidas, en los textos analizados del área de astronomía.

Los artículos científicos como nodos de una red, establecen relaciones con otros artículos a través de las referencias y las citas. En este ámbito, las referencias del artículo pueden desarrollar, desde el punto de vista semiótico, distintos interpretantes. En este caso nos referimos a dos: (1) las referencias como elementos de acreditación de los textos citantes, y (2) las referencias, reasignadas como citas, como elementos acreditantes de los textos citados. Para identificarlos utilizamos el análisis tríadico de los conceptos de la semiótica de Peirce (1992), Amozorrutia (2010) e Vidales-González, (2010). Se utilizan dos esquemas tríadicos: el primero se refiere al rol de la referencia como parte del artículo y el segundo a la referencia reasignada como cita Small (1978), Cronin (1981, 2000) e Collazo-Reyes (2012). El primer interpretante se desarrolla al momento de incluir referencias en una publicación, hechas a otros trabajos previamente publicados y, el segundo, cuando un trabajo aparece citado, después de su publicación, en otros trabajos. En 
ambos casos se identifican las direcciones de los flujos de comunicación desarrollados entre textos. En el caso de las referencias, identificamos los flujos que van hacia la literatura referida en tres contextos distintos: local, regional e internacional. En el caso de las citas los flujos también pueden proceder de contextos locales, regionales o internacionales.

Los resultados muestran que los contenidos de los trabajos publicados en el BOTT, forman parte de la red de relaciones internacionales de intertextualidad referencial entre textos (Durañona et al., 2006) aceptados en revistas cientificas y que, en este escenario, juegan roles de textos acreditados y de textos que acreditan, en un proceso mutuo de certificación de conocimientos seguido en el sistema de comunicación científica moderna. Estos aspectos son eventos emergentes, en el proceso histórico de formación del texto científico moderno en astronomía y le aportan novedades cualitativas a la estructura de los contenidos, las funciones de comunicación y el estilo del género científico en México.

\section{Métodos}

Se utilizó como fuente principal de análisis 154 textos completos de los trabajos publicados en el BOTT; las referencias hechas en el cuerpo de los trabajos, durante el periodo 1952-1972, y las citas recibidas en el periodo 1972-2013. Las referencias se recuperaron a través de una búsqueda en los contenidos de todos los textos. En el caso de las referencias incompletas, se consideraron las que incluían como información mínima el nombre del autor o la fuente y el año de publicación. Las citas fueron recuperadas del Web of Science (WoS), a través de la modalidad de búsqueda Cited Reference Search, por cada uno de los nombres de los autores participantes en los trabajos publicados en el BOTT. Se trata de citas hechas en revistas incluidas en el WoS para trabajos en el BOTT, que es una revista sin cobertura en este sistema de información. Las referencias y las citas fueron organizadas en archivos relacionales independientes, con una estructura de datos que incluye cinco campos de información: autor, año, fuente, volumen y página. Se desarrolló una base de datos relacional que incluye los 154 trabajos publicados en el BOTT, asociados a través de tablas relacionales, con los archivos de las referencias bibliográficas hechas en los trabajos y las citas recibidas.

\section{Modelo de análisis semiótico de las referencias y las citas}

El modelo semiótico de análisis tradicional consiste de tres elementos: signo o representamen (primeridad), objeto (segundidad) e interpretante (terceridad). El signo es la referencia bibliográfica que sustituye a un objeto físico que puede ser el registro bibliográfico, un artículo, capítulo, libro, o memoria. El interpretante tiene que ver con un concepto emergente de acreditación internacional de los textos en astronomía. Una novedad cualitativa en sus contenidos desarrollada a través de funciones de comunicación propias de las referencias y las citas. El desarrollo de este sentido de acreditación de los textos se explica a través de los modelos tríadicos de las Figuras 2 y 3.

\section{Resultados}

Encontramos un total de 1.139 referencias publicadas en 81 fuentes de distintos tipos. El 78,0\% corresponden a artículos en revistas incluidas en el WoS, el $12,0 \%$ a trabajos en fuentes no publicadas como conferencias, tesis, reportes, entre otros; el $6.5 \%$ se encuentran en fuentes publicadas pero que no son

\section{La referencias interpretadas como signo}

Análisis tríadico de C. Peirce

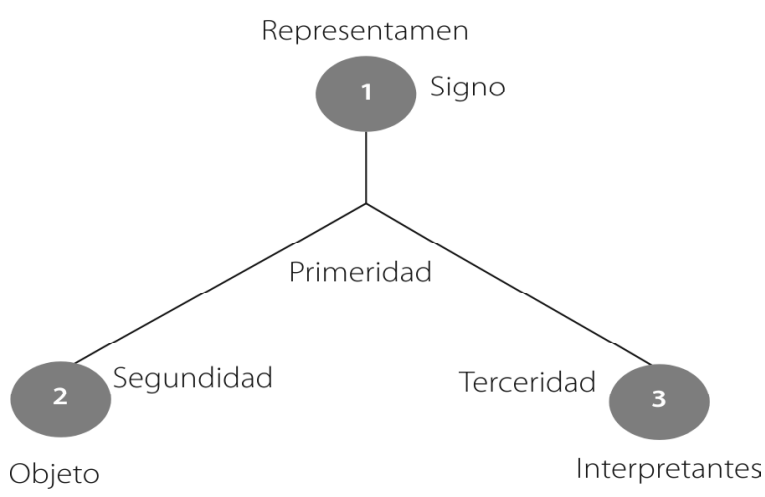

Figura 1. Modelo semiótico de análisis tríadico de C. Peirce.

Fuente: Elaboración propia, 2014 
cubiertas por el WoS. El 3.5\% restante se trata de referencias sin información sobre la fuente de publicación.

De los 154 trabajos publicados en el BOTT, 129 recibieron 2,405 citas en el periodo 1961-2013. Las citas proceden de trabajos publicados en 116 revistas, publicadas en distintos países, con una alta concentración de más del 80\% en revistas de United States of America. Cerca del $6 \%$ proceden de trabajos publicados en revistas (Revista Mexicana de Astronomíay Astrofísica) y memorias de congresos celebrados en México.

\section{Análisis semiótico de referencias}

En primer lugar, se analiza el proceso de significación de la referencia como un elemento de persuasión en la retórica del discurso científico (Restrepo Forero, 2004). Durante las distintas prácticas de construcción, arbitraje y publicación de los textos, las referencias desarrollan un interpretante de acreditación del estilo argumentativo de los contenidos. El nivel de primeridad (representamen), se refiere a la etapa de construcción de los textos. Cada vez que se utiliza una referencia bibliográfica se crea una relación de intertextualidad con otro texto, generalmente, ya publicado. En esta etapa el autor utiliza elementos de la referencia bibliográfica o de los textos citados, como convenciones de redacción, para representar el texto citado. Las prácticas de intertextualidad referencial utilizadas en el BOTT tienen las siguientes características: se utilizó, como signo principal de las referencias, números consecutivos dentro del texto en forma de super índices. Cada número apunta a una referencia

\section{Las referencias como interpretantes semióticos de persuasión}

Análisis tríadico

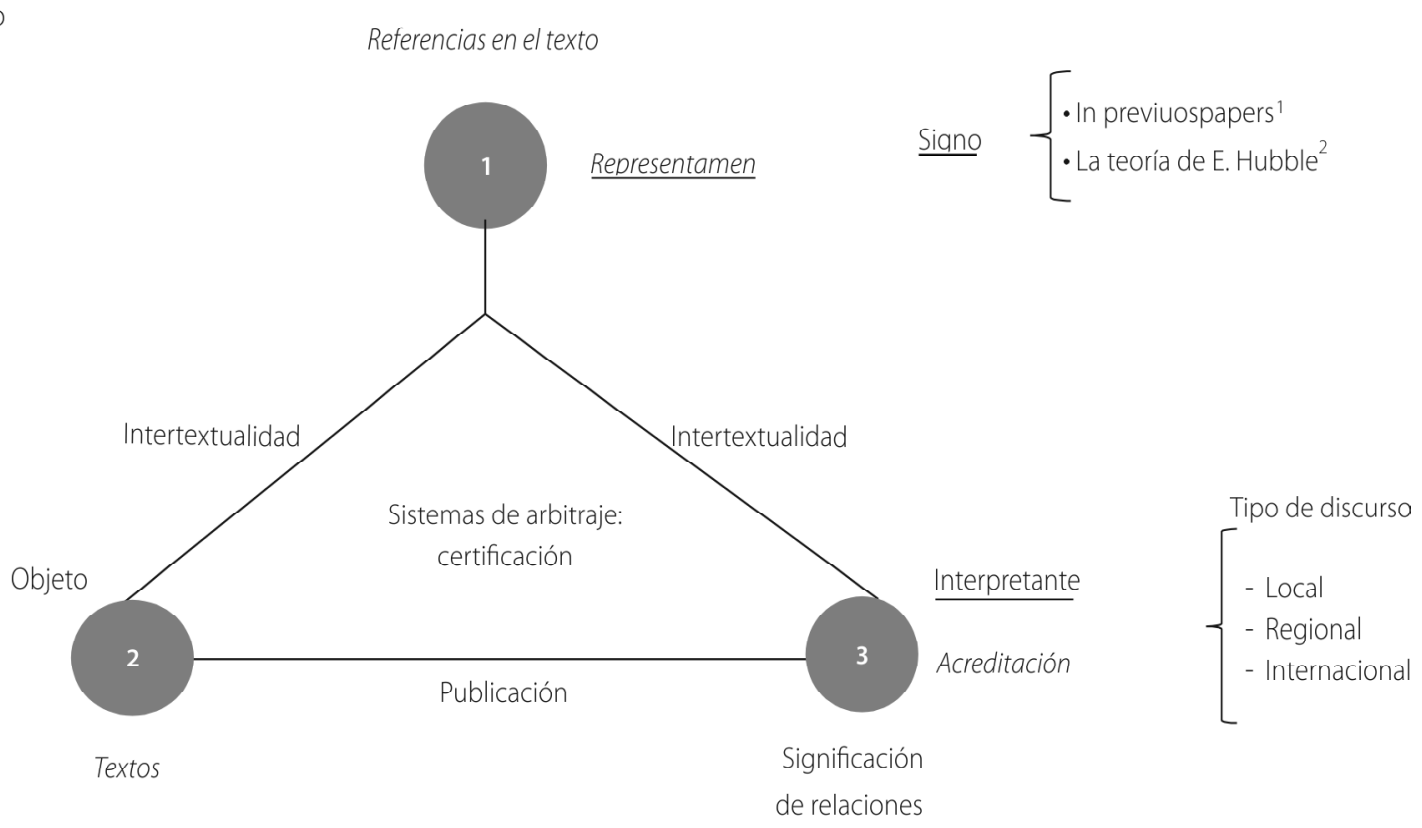

Flujos de comunicación

Referencias

1. W. J. Luyten. Ap. j. 58, 75, 1953. A. J. 61, 261, 1956.

2. Ap. J. vol 113, No. 3 November 1951.

Figura 2. Modelo de análisis tríadico de la referencia como interpretante semiótico de acreditación.

Fuente: Elaboración propia, 2014. 
bibliográfica de la obra citada, consignadas al final del texto en la sección de referencias. Para las notas de pie de página se utilizó como signo el asterisco en el texto y consignan aspectos diferentes a las referencias bibliográficas. En esta primera instancia del modelo, las referencias se reasignan como parte del contenido de los trabajos citantes, como elementos de apoyo al estilo argumentativo y de persuasión propios del género científico. Las referencias más comunes son para artículos en revistas internacionales, con un promedio de 7 referencias cada uno.

El segundo nivel (objetos) de la triada, se refiere a las referencias bibliográficas completas, organizadas como una unidad específica en la división de contenidos de los textos físicos, como objetos completos. Una sección que incluye un promedio de 7 referencias por documento, hechas a distintos tipos de fuentes que dividimos en cuatro tipos: (1) para artículos de revistas, no se incluye el título del trabajo, solamente el nombre del autor, título de la revista, volumen, página y año; (2) en el caso de libros, capítulos, tesis y congresos, se incluye el autor, título de la obra, lugar de edición, editorial y año; (3) cuando se hace referencia a los nombres de los autores del artículo, la referencia solo incluye el título de la revista, volumen, página y año; (4) en las referencias a las conversaciones privadas, datos no publicados y la correspondencia personal, se incluye el nombre del autor. Cada referencia puede desarrollar un rol distinto como fuente de acreditación del texto citante, de acuerdo con las siguientes características. El nivel de comunicación científica de pertenencia: local, regional o internacional; el tipo de investigación, teórica, experimental, difusión; el idioma de publicación; el prestigio de los autores en el campo de investigación; el tipo de revista; con o sin arbitraje, idioma, país de edición, y presencia en índices internacionales; actualidad de la información, identificada a partir del año de publicación. Estas características, actúan en favor o en contra de los textos escritos en los procesos de arbitraje y negociación de su publicación.

En el tercer nivel del modelo, los procesos de acreditación de los textos como objetos hechos públicos en revistas científicas, son eventos de comunicación que se repiten y generan circunstancias de producción de sentido. En este escenario de la comunicación y de acuerdo con el modelo de la Figura 1, los elementos que conforman el contenido de los textos del BOTT pueden desarrollar distintos interpretantes semióticos. En el caso que nos ocupa, cada referencia, en el texto, establece relaciones de intertextualidad con la literatura de algún tipo de discurso. Las relaciones pueden apuntar hacia la literatura y discursos considerados como periféricos, regionales o internacionales. En la medida que estas relaciones se repiten, con trabajos de un mismo discurso, las relaciones se distinguen y significan como flujos de comunicación entre textos con discursos afines. Estas relaciones se interpretan como eventos de acreditación del discurso de los documentos publicados en el BOTT en el discurso de los documentos referidos. En términos semióticos, este proceso de acreditación es un interpretante que desarrollan las referencias, como una función comunicativa que caracteriza su rol en el sistema de comunicación científica.

De este proceso de acreditación surgen distintos flujos de comunicación, mostrados en la Tabla 1 (A Referencias hechas). Cuando estas relaciones apuntan hacia la literatura del mismo país o de países de la misma región, tenemos acreditaciones de tipo endogámica y regional, respectivamente. Cuando se refieren a la literatura internacional tenemos acreditaciones en el discurso de corriente principal en el área de astronomía.

\section{Análisis semiótico de las citas}

En una etapa posterior a su publicación, los textos desarrollan un segundo interpretante de acreditación a través de las funciones de comunicación de las citas. Este sentido de las citas se construye a través de la misma relación tríadica de signo, objeto e interpretante. De acuerdo con el modelo de la Figura 3, en el primer nivel de la tríada, utilizamos la referencia no como parte del texto citante (según el modelo de la Figura 2). Nos interesa la referencia utilizada como signo del texto físico citado, representado por el objeto del segundo nivel de la tríada. Cuando un autor utiliza una referencia bibliográfica en alguna parte del contenido de su trabajo, las referencias se reasignan como citas y se establece una relación de comunicación, donde el documento que hace la cita es instanciado como documento citante y el que la recibe como documento citado. Cada vez que el objeto citado (segundo nivel de la tríada), es referido en 


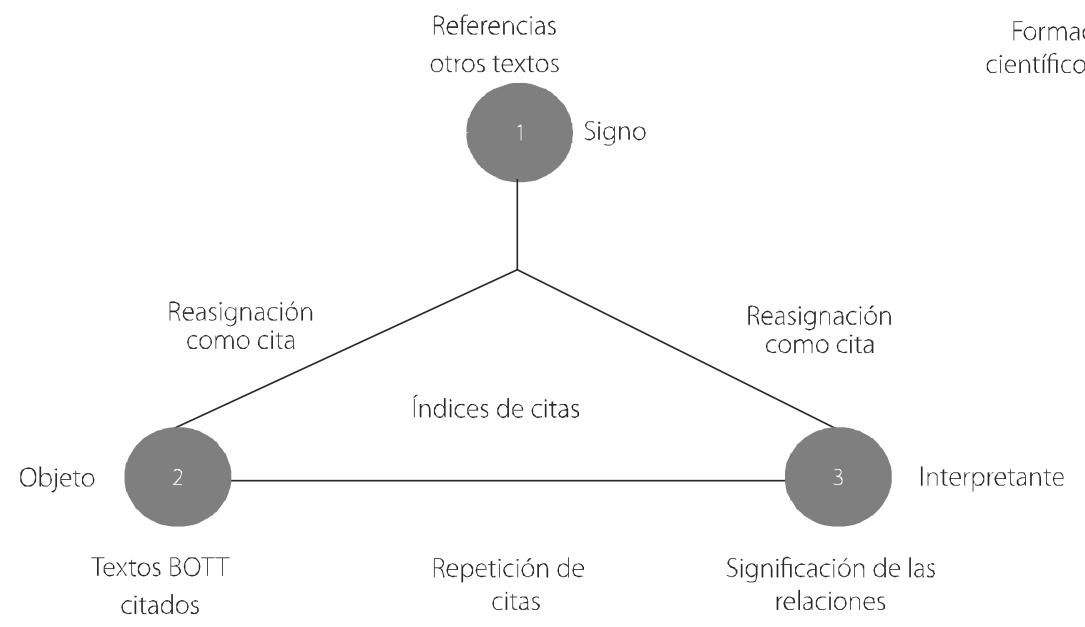

Formación del texto ientífico en astronomía

Figura 3. Análisis semiótico de la cita bibliográfica como elemento acreditante.

Fuente: Elaboración propia, 2014

Tabla 1. Flujos de comunicación científica resultantes de los trabajos publicados en el Boletín de los Observatorios de Tonantzintla y Tacubaya: A) referencias hechas, 1952-1972; B) citas recibidas, 1961-2013.

\begin{tabular}{|c|c|c|c|c|c|c|}
\hline \multicolumn{7}{|c|}{ A) Referencias hechas } \\
\hline Flujos & Autores Citantes & Revista & Revista Citada & Relaciones & $\%$ & Flujos de comunicación \\
\hline 1 & $L$ & BOTा & $E$ & 885 & 77.7 & Local-local-externos \\
\hline 2 & $L$ & BOTा & L & 69 & 6.06 & Endógenos \\
\hline 3 & $E$ & BOTा & $E$ & 158 & 13.87 & Externo-local-externo \\
\hline 4 & $E$ & BOTा & L & 18 & 1.58 & Externo-local-local \\
\hline 5 & $\mathrm{R}$ & BOTा & $E$ & 6 & 0.53 & Regional-local-externo \\
\hline 6 & $\mathrm{R}$ & BOTT & $\mathrm{L}$ & 3 & 0.26 & Regional-local-local \\
\hline \multicolumn{7}{|c|}{ B) Citas recibidas } \\
\hline Flujos & Autore Citante & Revista & Revista Citada & Relaciones & $\%$ & Flujos de comunicación \\
\hline 1 & $L$ & $\mathrm{~L}$ & $\mathrm{~L}$ & 109 & 4.53 & Endógenos \\
\hline 2 & $L$ & $E$ & $L$ & 234 & 9.73 & Local-externo-local \\
\hline 3 & E & E & $\mathrm{L}$ & 1998 & 83 & Externo-externo-local \\
\hline 4 & E & $L$ & $\mathrm{~L}$ & 46 & 1.91 & Externo-local-local \\
\hline 5 & $\mathrm{R}$ & E & $\mathrm{L}$ & 18 & 0.75 & Regional-externo-local \\
\hline
\end{tabular}

Nota: $L=$ local; $E=$ externo; $R=$ regional.

Fuente: Elaboración propia, 2014.

algún tipo de literatura publicada, se considera como un signo indicativo de acreditación del objeto citado en el discurso de la literatura del objeto citante. De acuerdo con el tercer nivel de la tríada, los textos del BOTT, desarrollan un interpretante de textos acreditantes en el sistema de comunicación científica, cada vez que son citados por otros textos. A diferencia de las referencias, aquí las citas se interpretan como eventos acreditantes, donde los textos del BOTT funcionan como elementos de apoyo a la retórica argumentativa y de persuasión de los textos citantes. Así, en la medida que la procedencia de las citas se repiten, con trabajos pertenecientes a un 
mismo discurso (local, regional, internacional), las relaciones se distinguen y significan como flujos de comunicación entre textos de discursos afines. Estos flujos traducidos a estructuras de comunicación, se utilizan como testimonios del proceso que certifica el discurso de los documentos citados en el discurso de los documentos citantes. El interpretante semiótico de las citas acredita el discurso de los documentos citados en distintos tipos de literatura que se muestran en la Tabla 1.

La Tabla 1 (B-Citas recibidas), muestra cinco flujos de comunicación resultantes de las citas hechas a los trabajos del BOTT. Incluye flujos endógenos, localexternos y externos-locales, principalmente.

$$
L=\text { local; } E=\text { externo; } R=\text { regional }
$$

La Tabla 1 (A) referencias hechas, muestra seis flujos resultantes de las referencias hechas en los trabajos del BOTT. Un poco más de tres cuartas partes (77.7\%) de las referencias fueron hechas por autores adscritos a instituciones locales para trabajos publicados en revistas extranjeras. La Tabla 1 (B), muestra los títulos de las revistas citadas y el número de referencias. Cerca de la mitad de las referencias fueron para trabajos publicados en Astrophysical Journal. Los autores adscritos a instituciones externas que publicaron en el BOTT realizaron cerca del 15\% de las referencias. Las referencias hechas por autores locales a trabajos publicados en fuentes locales, principalmente el BOTT, representan el 6\% y se denominaron como flujos endógenos. Los autores regionales, adscritos a instituciones de países de América Latina, realizaron menos de 10 citas.

De acuerdo con la Tabla 1 (B Citas recibidas), un poco más de cuatro quintas partes (83\%) de las citas fueron hechas por autores adscritos a instituciones extranjeras en trabajos publicados en fuentes internacionales. El segundo flujo más importante de citas, procede de autores locales que publican en revistas externas. También existen flujos endogámicos de acreditación entre autores, trabajos y fuentes locales. Los autores regionales, adscritos a instituciones de países de América Latina, realizaron menos de 18 citas en revistas externas.

De acuerdo con estos flujos procedentes, principalmente, de autores y revistas extranjeras, los trabajos del BOTT están acreditados en el mismo sistema de información de los trabajos y las revistas citantes, que es el del discurso dominante en astronomía a nivel internacional.

\section{Revistas citadas y citantes}

De acuerdo con las fuentes citadas de la Tabla 2 (A), cerca de la mitad de las referencias fueron hechas a trabajos publicados en Astrophysical Journal, una de las revistas de mayor prestigio en el campo. El 10\% de las referencias fueron para trabajos publicados en el propio BOTT que aparece en segundo lugar. Las referencias para libros y tesis suman cerca de un 4\%. Las fuentes de publicación citadas son editadas en distintos países (USA, México, Gran Bretaña, Japón, Holanda, Francia, Alemania), sin embargo, más de las tres cuartas partes de las referencias son para trabajos publicados en fuentes editadas en USA. De acuerdo con estos flujos repetitivos de intertextualidad referencial, los trabajos del BOTT están acreditados, principalmente, en trabajos y revistas del discurso dominante a nivel internacional.

La Tabla 2 (B fuentes citantes), muestra los títulos de las revistas citantes y el número de citas. El 85\% de las citas están hechas en los primeros 11 títulos de revistas. Nueve de estas, están publicadas en USA. Se destacan los dos primeros títulos Astrophysical Journal y Astronomy \&Astrophysics, que suman más de mil citas, cerca del 50\% del total. El 12\% de las citas proceden de trabajos publicados en una revista de la Gran Bretaña (Monthly Notices of the Royal Astronomical Society), ubicada en tercer lugar. Las revistas locales, lugares 7 (Revista Mexicana de Astronomía y Astrofísica) y 9 (Revista Mexicana de Astronomía y Astrofísica, Serie de Conferencias), realizaron el $5 \%$ de las citas. Los títulos de las revistas ubicadas en los lugares 1, 3 y 4 de las Tablas 2 (A) y 2 (B), respectivamente, son las mismas. Esto es señal de una importante correlación entre las revistas que acreditan los trabajos del BOTT, tanto a través de las referencias como a través de las citas.

\section{Discusión y Conclusión}

Las prácticas de investigación científica desarrolladas en los laboratorios de Tonantzintla y Tacubaya, generaron un espacio de encuentro entre componentes de una práctica científica: especialistas en 
Tabla 2. Intertextualidad referencial resultante de los trabajos publicados en el Boletín de los Observatorios de Tonantzintla y Tacubaya: A) Fuentes citadas, 1952-1972; B) fuentes citantes, 1961-2013.

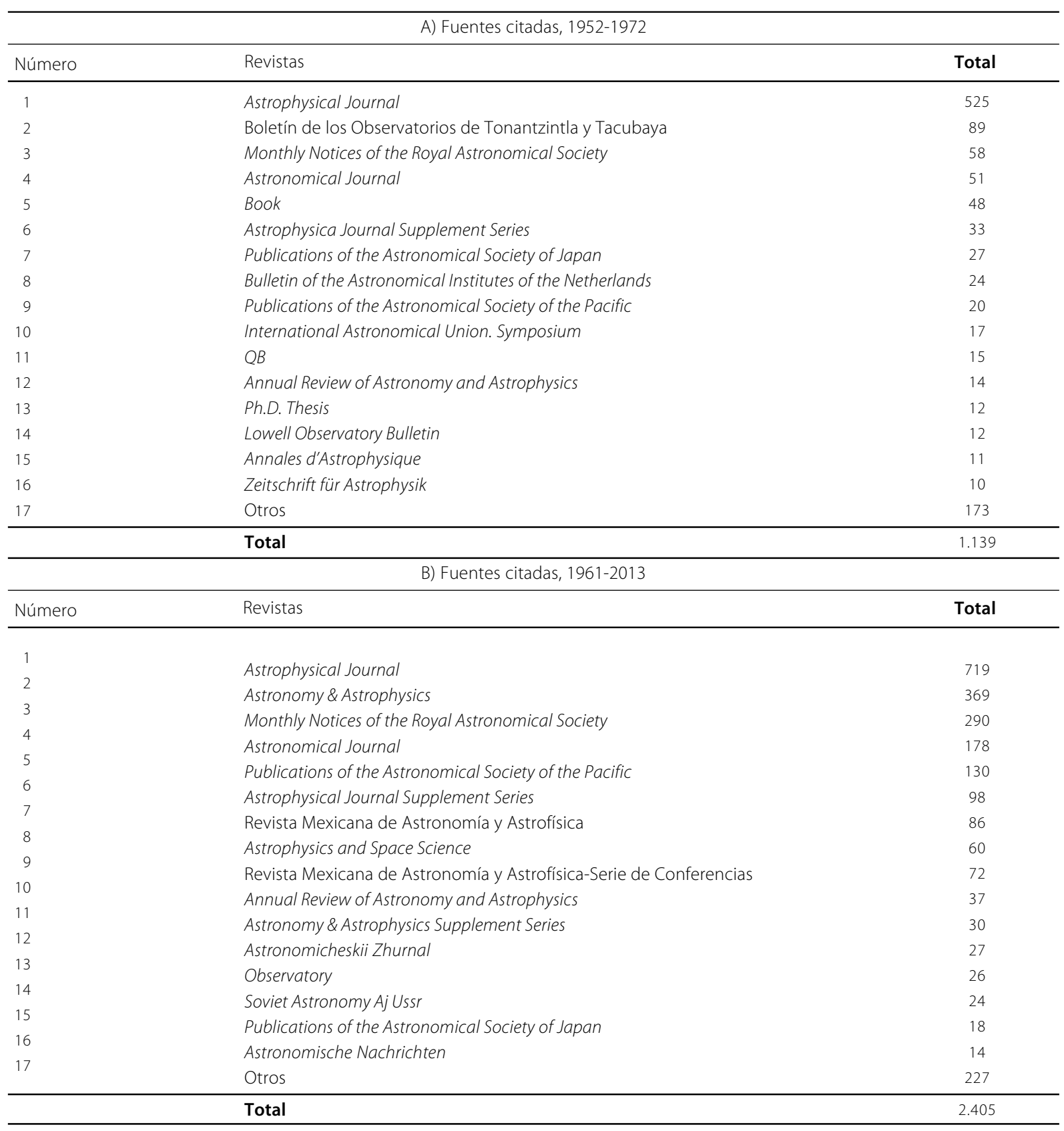

Fuente: Elaboración propia, 2014.

el área de astronomía, metodologías, prácticas de investigación, instrumental científico, conocimientos y dominio de los paradigmas científicos, y una fuente de difusión de los resultados de investigación. La coincidencia de estos elementos, se interpretan como aspectos determinantes, en la medida que incidieron en el desarrollo de circunstancias estables que hicieron posible la construcción de prácticas científicas productivas en el área de astronomía. Esta condición de estabilidad, permitió la continuidad en la producción de resultados de investigación y la formación de estructuras de comunicación científica moderna. 
El análisis tríadico de la semiótica, como proceso de significación de sentido, es un recurso de utilidad para identificar distintos interpretantes que desarrollan las referencias y las citas, como funciones de comunicación en los textos. Estos interpretantes ayudan a caracterizar aspectos emergentes en el proceso de formación que sigue el género científico en cualquier país; como parte de una dinámica que acompaña la conformación de las estructuras de los sistemas de comunicación científica en formación, a cualquier nivel, local, regional o internacional.

Los resultados muestran una importante correlación entre los títulos de las revistas citadas y citantes, donde el punto de encuentro son los textos del BOTT. Esto significa que los contenidos de los trabajos publicados en el Boletín, forman parte de la red de relaciones internacionales de intertextualidad referencial entre textos aceptados en las revistas científicas más importantes en el área de astronomía: Astrophysical Journal; Astronomy \& Astrophysics; Monthly Notices of the Royal Astronomical Society; Astronomical Journal; Publications of the Astronomical Society of the Pacific, entre otras. En este escenario de medios de difusión, donde se construye el discurso dominante del área, las publicaciones del Boletín juegan roles de textos acreditados y que acreditan, o acreditantes; formando parte de un proceso mutuo de certificación de conocimientos seguido en el sistema de comunicación científica moderna. Estos aspectos, son eventos emergentes en el proceso histórico de formación del texto científico moderno en México. Aportan novedades cualitativas, al estilo argumentativo del género científico en el área de astronomía. Las novedades incluyen marcas del contexto local e internacional que trascienden los patrones endogámicos del sistema de comunicación científica local, dependiente de mecanismos endogámicos de acreditación de conocimientos y, tradicionalmente, basado en fuentes de difusión doméstica, idioma local y un estilo argumentativo escaso de recursos de intertextualidad referencial.

\section{Agradecimientos}

Al Consejo Nacional de Ciencia y Tecnología - Red de Complejidad, Ciencia y Sociedad.

\section{Referencias}

Abt, H.A. Symposium summary. Revista Mexicana de Astronomía y Astrofísica, v.39, p.117-118, 2011 a.

Abt, H.A. The Tonantzintla search for high luminosityStars. Revista Mexicana de Astronomía y Astrofísica, v.39, p.65-68, 2011b.

Amozorrutia, J.A. De la semiótica a la cibernética: una estrategia para modelar sistémicamente el sentido. Razón y Palabra, v.15, n.72, p.1-24, 2010.

Bartolucci, J. Formación tardía de comunidades científicas: el caso de los astrónomos mexicanos. Quipu, v.8, n.3, p.361-377, 1991.

Bartolucci, J. La modernización de la ciencia en México: el caso de los astrónomos. México: UNAM, 2000.

Bazerman, C. Making references: Empirical contexts, choices, and constraints in the literary creation of the compton effect. In: Bazerman, C. Shaping written knowledge: The genre and activity of the experimental article in science. Madison: University of Wisconsin Press, 1988a. p.187-234.

Bazerman, C. Shaping written knowledge: The genre and activity of the experimental article in science. Madison: University of Wisconsin Press, 1988b, 356p.
Bazerman, C. Intertextual self-fashioning: Gould and Lewontin's representations of the literature. In: Selzer, J. (Ed.). Understanding scientific prose. Madison: University of Wisconsin Press, 1993. p.20-41.

Beuchot, M. La semiótica: teoría del signo y el lenguaje en la historia. México: Fondo de Cultura Económica, 2004.

Bok, B.J. Mexican astronomy, 1930-1950. Revista Mexicana de Astronomía y Astrofísica, v.7, (Esp.), p.21-25, 1983.

Bornmann, L.; Daniel, H.D. What do citation counts measure? A review of studies on citing behavior. Journal of Documentation, v.64, n.1, p.45-80, 2008.

Carmona Sandoval, J.C. Discurso y artículo científico: una aproximación retórica. Raximhai, v.9, n.1, p.117-152, 2013.

Collazo-Reyes, F. Modelo teórico metodológico para analizar la visibilidad internacional de las ciencias físicas en América Latina y el Caribe. 2012. Tesis (Doctorado em Bibliotecología y Estudios de la Información). Universidad Nacional Autónoma de México, México, 2012.

Cornejo-Rodríguez, A. The first years of optics in México and the rol of Boletín de los Observatorios de Tonantzintla y Tacubaya on its development. Revista Mexicana de Astronomía y Astrofísica, v.39, p.109-115, 2011. 
Cozzens, S.E. What does citations count? The rhetoric-first model. Scientometric, v.15, n.5, p.1437-447, 1989.

Cronin, B. The need for a theory of citation. Journal of Documentation, v.37, n.1, p.16-24, 1981.

Cronin, B. The citation process: The role and significance of citation in Scientific Communication. London:Taylor Graham, 1984.

Cronin, B. Semiotic and evaluative bibliometrics. Journal of Documentation, v.56, n.4, p.440-453, 2000.

Cueto, M. Science under adversity: Latin American Medical Research and American Private Philantrophy, 1920-1960. Minerva, v.35, n.3, p.233-245, 1997.

Davis, P.M. Reward or persuasion? The battle to define the meaning of a citation. Learned Publishing, v.22, n.1, p.5-11, 2009 .

Durañona, M.A. et al. Textos que dialogan: la intertextualidad como recurso didáctico. Madrid: Comunidad de Madrid, 2006.

Gallo, J. Magnetic work of the National Astronomical Observatory of the National University of México. Transactions American Geophysical Union, v.20, n.3, p.394, 1939.

Gilbert, G.N. Referencing as persuasion. Social Studies of Science, v.7, p.113-122, 1977

González-Tapia, F.B.; Muñoz-García, M.L. Estudio históricobibliométrico de la literatura científica generada en astronomía en México, durante el siglo XX. 2013. Tesis (Licenciatura en Biblioteconomía). Escuela Nacional de Biblioteconomía y Archivonomía, Ciudad de México, México, 2013.

Haberer, A. Intertextuality in theory and practice. Literature, v.49, n.5, p.54-67, 2007.

Knorr-Cetina, K. The manufacture of knowledge: An essay on the constructivist and contextual nature of science. Oxford: Pergamon Press, 1981

Latour, B. Science in action: How to follow scientists and engineers through society. Cambridge, MA: Harvard University Press, 1987

López-Cruz, O. Informal introduction. Revista Mexicana de Astronomía y Astrofísica, v.39, p.xiii-xiv, 2011.
Luna-Morales, M.E. et al. Early patterns of scientific production by mexican researchers in mainstream journals, 1900-1950. Journal of the Americam Society Information Science and Technology, v.60, n.7, p.1337-1348, 2009.

MacRoberts, M.H.; MacRoberts, B.R. Testing the Ortega hypothesis: Facts and artifacts. Scientometrics, v.12, n.5-6, p.293-295, 1987.

Merton, R.K. The Matthew effect in science. Science, v.159, n.5, p.56-63, 1968.

Moed, H.F. What do references and citations measure? In: Moed, H.F. (Ed.). Citation analysis research evaluation. Netherlands: Springer, 2005. p.193-208.

Munch, G. Model Solar Atmospheres. Astronomical Journal, v.106, n.2, p.217-223, 1947.

Paul, D. In citing chaos: A study of the rhetorical use of citations. Journal on Bussines Technology Communication, v.4, n.2, p.185-216, 2000.

Peirce, C.S. The essential Peirce: Selected philosophical writings (1867-1983). Bloomington: Indiana University Press, 1992.

Pismis, P. La astronomía en México. Hacia su etapa actual. Revista Mexicana de Astronomía y Astrofísica, v.14, n.1, p.35-42, 1987.

Porter, J. E. Intertextuality and the discourse community. Rhetoric Review, v.5, n.1, p.35-47, 1986.

Restrepo Forero, O. Retórica de la ciencia sin "retórica": sobre autores, comunidades y contextos. Revista Colombiana de Sociología, v.23, p.251-268, 2004.

Rivera-Terrazas, M. The program of the Tonantzintla Schmidt camera. Astronomical Journal, v.55, n.3, p.65, 1950

Small, H.G. Cited documents as concept symbols. Social Studies of Science, v.8, n.3, p.327-340, 1978.

Torres-Peimbert, S.; López-Cruz, O.; Peimbert, M. Historical note. Revista Mexicana de Astronomía y Astrofísica, v.39, p.vi-vii, 2011.

Vidales-González, C. Semiótica y teoría de la comunicación. Tomo I. México: CECyTE, 2010, 135p.

White, H.D. Reward, persuasión, and the Sokal Hoax: A study in citation identities. Scientometrics, v.60, n.1, p.93-120, 2004. 
